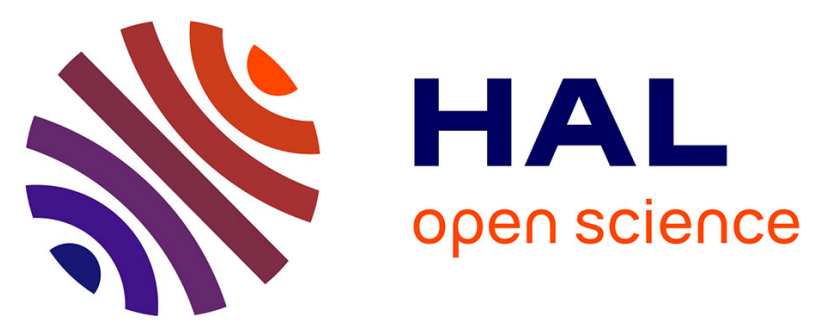

\title{
Core-Shell Gold/Silver Nanoparticles for Localized Surface Plasmon Resonance-Based Naked-Eye Toxin Biosensing
}

Alexis Loiseau, Lu Zhang, David Hu, Michèle Salmain, Yacine Mazouzi, Raphaël Flack, Bo Liedberg, Souhir Boujday

\section{To cite this version:}

Alexis Loiseau, Lu Zhang, David Hu, Michèle Salmain, Yacine Mazouzi, et al.. Core-Shell Gold/Silver Nanoparticles for Localized Surface Plasmon Resonance-Based Naked-Eye Toxin Biosensing. ACS Applied Materials \& Interfaces, 2019, 11 (50), pp.46462-46471. 10.1021/acsami.9b14980 . hal-02427513

\section{HAL Id: hal-02427513 \\ https://hal.science/hal-02427513}

Submitted on 7 Dec 2020

HAL is a multi-disciplinary open access archive for the deposit and dissemination of scientific research documents, whether they are published or not. The documents may come from teaching and research institutions in France or abroad, or from public or private research centers.
L'archive ouverte pluridisciplinaire HAL, est destinée au dépôt et à la diffusion de documents scientifiques de niveau recherche, publiés ou non, émanant des établissements d'enseignement et de recherche français ou étrangers, des laboratoires publics ou privés. 


\title{
Core-shell Gold Silver Nanoparticles for LSPR-Based Naked-Eye Toxin Biosensing
}

\author{
Alexis Loiseau ${ }^{1, \ddagger}$, Lu Zhang ${ }^{1,2,3, \ddagger}$, David Hu${ }^{1}$, Michèle Salmain², Yacine Mazouzi ${ }^{1}$, Raphaël Flack ${ }^{1}$, Bo \\ Liedberg ${ }^{3, *}$, Souhir Boujday ${ }^{1, *}$ \\ ${ }^{1}$ Sorbonne Université, CNRS, Laboratoire de Réactivité de Surface (LRS), 4 place Jussieu, F 75005 Paris, France. \\ ${ }^{2}$ Sorbonne Université, CNRS, Institut Parisien de Chimie Moléculaire (IPCM), 4 place Jussieu F 75005 Paris, France. \\ ${ }^{3}$ Centre for Biomimetic Sensor Science, School of Materials Science and Engineering, Nanyang Technological University, \\ Singapore 637553.
}

KEYWORDS: Plasmonic Nanoparticles, LSPR, Gold/Silver Core-Shell, Surface Functionalization, Biotoxin Detection, Naked-Eye Readout, Biosensors

\begin{abstract}
The Localized Surface Plasmon Resonance (LSPR) phenomenon provides a versatile property for biodetection. Herein, this unique feature was employed to build up a homogeneous optical biosensor to detect staphylococcal enterotoxin A (SEA) in solution down to very low levels by naked-eye readout. If the initial position of the LSPR band is located in the cyan region, even a small red shift $(\sim 2-3 \mathrm{~nm})$ induced by Refractive index (RI) change close to the surface of nanoparticles (NPs), could make the light absorption transit from cyan to green and become visually detectable via a concomitant change in the complementary colors. In this work, we aimed at synthesizing two types of NPs based on compositionally complex core-shell NPs - Ag shell on AuNPs (Au@AgNPs) and Ag inside gold nanoshell (Ag@AuNPs). By controlling the thickness of the shells as well as their surface chemistry with anti-SEA antibody, the LSPR band was tuned to near 495 and 520 nm, for Ag@AuNPs and Au@AgNPs, respectively. The two particle systems were subsequently applied as transducers to spectroscopically and visually detect anti-SEA antibody - SEA interactions. Upon addition of SEA large red-shifts of the LSPR band were observed spectroscopically and the limits of detection (LOD) were estimated to $0.2 \mathrm{nM}$ and $0.4 \mathrm{nM}$ for the Au@AgNPs and Ag@AuNPs, respectively. Although the two sets of NPs gave almost identical LOD, the Ag@AuNPs whose initial position of the LSPR band was tuned in the cyan to green region $(\sim 500 \mathrm{~nm})$ displayed a substantially more distinct color change from orange to red, as revealed by the by naked-eye. We foresee significant potential to this strategy in medical diagnostic and environmental monitoring, especially when basic laboratory infrastructure is sparse or non-existent.
\end{abstract}

\section{INTRODUCTION}

Plasmonic metal nanoparticles (NPs) have shown a great potential for chemical and biological sensor applications over the past decades. ${ }^{1-5}$ This is mainly due to their unique optical properties originating from localized surface plasmon resonance (LSPR) phenomenon, which is responsible for the bright color of the NPs suspensions. ${ }^{6-8}$ This feature led to the design of numerous homogeneous and solid-phase schemes enabling naked-eye detection of analytes. Indeed, plasmonic NPs are the most commonly used labels for lateral-flow assays reported in the literature. ${ }^{9}$ For homogeneous assays, the most common format allowing for naked-eye readout relies on aggregation/dispersion phenomena: plasmonic particles aggregation results in a distinct red shift of the LSPR band and to a color change from red to blue. Since the introduction of this format by Mirkin et al. ${ }^{1}$ it has been extensively applied for the detection of various targets. ${ }^{10}$ Other strategies based on etching and/or growth of plasmonic particles have been designed during the last decades to allow for a visual reading of the biosensing events (see reference ${ }^{11}$ and references therein). Apart from these colorimetric assays, plasmonic NPs are widely utilized as LSPR-biosensors using UV-Visible spectrometers as transducers. Indeed, the position of the LSPR band is very sensitive to local changes of the refractive index (RI) and can be correlated to the changes in chemical and/or physical environment near the NPs surface. ${ }^{12-13}$ In 1998, Englebienne reported the first use of gold nanoparticles to monitor antibody/antigen recognition. ${ }^{14}$ These LSPR-biosensors exploiting local RI changes led to extremely sensitive devices capable of detecting LSPR band shifts at the nm level and applicable to numerous targets. ${ }^{15-16}$ However, this sensitivity can only be achieved using a UV-Vis spectrometer as the color change induced by such small shifts in the wavelength is not observable by the naked-eye. In a recent study, ${ }^{17}$ we highlighted the importance of tuning the optical properties of noble metal NPs to enable sensitive detection of minute optical transitions (wavelength shifts) by the naked-eye. This work showed that by tuning the NP composition and thereby the optical extinction to the cyan-green range (just below $500 \mathrm{~nm}$ ) it is possible to overlap with the sensitivity function of our vision system and locate the transducer response in a region displaying a sharp transition in optical response. As a consequence, an LSPR shift as small as $2 \mathrm{~nm}$ could be observed by the naked-eye. ${ }^{17}$ Locating the LSPR band in this region is possible by combining $\mathrm{Au}$ and $\mathrm{Ag}$ within the nanoparticle.

Among all the plasmonic NPs, gold and silver NPs (AuNPs and AgNPs, respectively) have attracted great attention for biosensing platform development due to their extremely high extinction coefficient in the visible spectral range. ${ }^{3,12,18-20}$ Spherical AuNPs and AgNPs display LSPR bands in the green and blue regions of the visible spectrum, respectively, ${ }^{21}$ which can be tuned by varying their size and shape. ${ }^{22-23}$ For small spherical AuNPs (of an average diameter of about $10 \mathrm{~nm}$ ), the LSPR band of the red-colored NPs suspensions is around $520 \mathrm{~nm}$, while for spherical AgNPs with the same average diameter, the absorption band is around $395 \mathrm{~nm}$, making the suspensions yellow-colored.5, 20, 24 Even if AuNPs remain the most 
studied system for biosensors because of their excellent chemical stability and biocompatibility, ${ }^{25-26}$ AgNPs offer improved extinction coefficients and a higher sensitivity to RI than AuNPs for a given size and shape. ${ }^{13,24}$ As an example, the optical response (LSPR shift) due to the addition of avidin to biocytin-coated metallic spherical NPs of similar size, was five times higher for AgNPs than for AuNPs. ${ }^{24}$ However, AgNPs might have a toxic effect in biological systems. They are also less stable under identical chemical conditions and barely reusable, which makes their use in repeated cycles more difficult than AuNPs. ${ }^{27-28}$ Mixing $\mathrm{Au}$ and Ag to form either alloys or core-shell AgAuNPs leads to a hybrid LSPR band that can be tuned continuously from 400 to $520 \mathrm{~nm}$ via changing the $\mathrm{Au} / \mathrm{Ag}$ ratio. ${ }^{29}$ Yet, to date, very few studies have involved mixed Au/Ag NPs for RI-based LSPR biosensing. Works dealing with the streptavidin/biotin couple as a model of biomolecular interaction were reported. ${ }^{30-31}$ Other examples such as sulfide detection, ${ }^{32}$ and antibiotics SERS aptasensing ${ }^{33}$ and, more recently, colorimetric detection of $\mathrm{DNA}^{34}$ and illicit drugs ${ }^{35}$ were described. However, to the best of our knowledge, mixed Au/Ag NPs plasmonic NPs have never been used for LSPR-immunosensing of a bio-toxin.

As the surface chemistry of mixed Au-Ag alloys is difficult to master, the core-shell structure is preferred to engineer mixed Au/Ag NPs and leads to well-distributed colloids in size and shape. ${ }^{36-37}$ Au-Ag core-shell nanoparticles (Au@AgNPs) were first synthesized by Morriss and Collins, ${ }^{38}$ and have since received great attention for plasmonic applications. ${ }^{5,18,34-35,}$ 39 This configuration, Au@AgNPs, where a gold core is coated by a silver shell is quite simple to achieve by growing a layer of Ag on previously prepared AuNPs and offers a homogeneous size distribution. ${ }^{40}$ Synthesizing Ag-Au core-shell NPs (Ag@AuNPs) is less straightforward. Indeed, when gold salt is added to a suspension containing AgNPs, hollow gold nanoshells (AuNS) are formed by galvanic replacement. ${ }^{41-43}$ This is due to the lower redox potential of the $\mathrm{Ag}^{+} / \mathrm{Ag}^{\mathrm{C}} \mathrm{couple}$ compared to the $\mathrm{Au}^{3+} / \mathrm{Au}$ couple $\left(0.80 \mathrm{~V} / \mathrm{SHE}\right.$ vs $0.99 \mathrm{~V} / \mathrm{SHE}$ for $\mathrm{Ag}^{+} / \mathrm{Ag}$ and $\mathrm{Au}^{3+} / \mathrm{Au}$, respectively). ${ }^{44}$ Although some papers describe gold growth on silver NPs, the resulting core-shell particles have imperfections, mainly holes, and sometimes complete removal of Ag core; TEM images of the so-synthesized Ag@AuNPs often show brighter central and darker outer nanostructure. ${ }^{45-47}$

Recently, we introduced an original pathway to form Ag-Au core-shell NPs (Ag@AuNPs) from AuNS. ${ }^{48}$ Porous AuNS were prepared by galvanic replacement from AgNPs, and we showed that the nanoconfinement effect caused pH-triggered silver reduction to preferentially occur at the inner walls of AuNS until a solid Ag core is formed inside the AuNS to form Ag@AuNPs. ${ }^{48}$ The presence of Au at the external sphere of Ag@AuNPs leads to a better stability of the colloids as mentioned previously and also allow for the use of gold chemistry to engineer bioconjugates that we showed to be stable over a year of storage at $4^{\circ} \mathrm{C}$ with preserved analytical performances. ${ }^{20}$ In addition, the synthesis method of Ag@AuNPs allows for a precise control of the amount of Ag within the shell and we were able to precisely tune the position of the LSPR band and locate it in the desired spectral region for naked-eye biosensing applications. ${ }^{48}$

In this study, we investigate two configurations of plasmonic core-shell nanoparticles: Au@AgNPs and Ag@AuNPs for LSPR-based naked-eye biosensing. The optical properties of these nano-objects were tuned to the cyan-green region by changing the silver shell thickness on the gold core for Au@AgNPs or by filling the inner volume in the hollow gold nanoshell particles Ag@AuNPs with silver. These plasmonic core-shell NPs were modified to engineer antibody-conjugated Au@AgNPs and Ag@AuNPs to be used as transducers for naked-eye immunosensing of a harmful biotoxin; staphylococcal enterotoxin A (SEA). For several years, our group has been especially interested in the detection of SEA. ${ }^{20,49-51}$ SEA is a small monomeric protein $(\mathrm{Mw}=28 \mathrm{kDa})$ with high thermal and proteolytic stability. ${ }^{52}$ It is the most commonly encountered biotoxin involved in staphylococcal food poisoning outbreaks. ${ }^{53}$ Ingestion of raw or even cooked food contaminated by SEA induces severe gastroenteritis, sometimes fatal to humans. ${ }^{20,50,54}$ In previous works, we mastered the engineering of stable antibody-AuNPs bioconjugates by covalent attachment of anti-SEA antibodies to spherical AuNPs to afford an immunoprobe to detect SEA. ${ }^{20,50-51}$ We then set up a homogeneous, solution-based assay, for which binding of SEA in the ng/mL range resulted in a red-shift of the LSPR band of the immunoprobe, only measurable by a dedicated instrument. ${ }^{20}$ Using the newly developed Ag@AuNPs we aim at achieving a naked-eye readout of the immunorecognition. The nanomaterials synthesized here were carefully characterized using a suite of highly sophisticated surface analytical techniques such as Transmission Electron Microscopy (TEM and STEM), X-EDS, UV-Vis spectroscopy, Dynamic Light Scattering (DLS), and zeta potential (ELS) measurements. The resulting bioconjugates were then used for SEA biosensing, and their analytical performances in terms of sensitivity and their limits of detection (LOD) were measured prior to their use for naked-eye readout of the biosensing event. We foresee significant potential of this strategy in medical diagnostic and environmental monitoring, especially in situations where basic laboratory infrastructure is sparse or non-existent.

\section{EXPERIMENTAL SECTION}

Materials. Hydrogen tetrachloroaurate(III) trihydrate $\left(\mathrm{HAuCl}_{4} .3 \mathrm{H}_{2} \mathrm{O} ; \geq 99.99 \%\right)$ was purchased from Alfa Aesar. Sodium citrate dihydrate $\left(\mathrm{Na}_{3} \mathrm{C}_{6} \mathrm{H}_{5} \mathrm{O}_{7} .2 \mathrm{H}_{2} \mathrm{O} ; \geq 99 \%\right)$, tannic acid $\left(\mathrm{C}_{76} \mathrm{H}_{52} \mathrm{O}_{46}\right)$, sodium borohydride (98\%), hydroxylamine hydrochloride (99\%), sodium hydroxide, bovine serum albumin (BSA), 2-iminothiolane hydrochloride (Traut's reagent: $\mathrm{C}_{4} \mathrm{H}_{7} \mathrm{NS} \mathrm{HCl}_{\text {; }}$ $\geq 98 \%$ ) and staphylococcal enterotoxin A (SEA) were purchased from Sigma Aldrich. Silver nitrate $\left(\mathrm{AgNO}_{3} ; \geq 99 \%\right)$ was purchased from Honeywell, Fluka. Hydrochloric acid (37\%) and nitric acid (68\%) were purchased from VWR chemicals. Affinity purified rabbit polyclonal anti-SEA antibody (Ab, reference in toxin technology catalog: LAI101) was purchased from Toxin Technology (Sarasota, FL, www.toxintechnology.com). All glassware was cleaned in a bath of freshly prepared aqua regia solution, and then rinsed thoroughly with Milli- $Q$ water before use.

Au-Ag core-shell nanoparticle preparation (Au@AgNPs). Colloidal AuNPs were synthesized according to the citrate/tannic acid (TA) reduction method adapted from ref. ${ }^{55-56}$ and optimized in ref. ${ }^{20,51} 100 \mathrm{~mL}$ of citrate-coated AuNPs 
were prepared from two stock solutions expected to afford particles with an average size of $13.5 \mathrm{~nm}$ (Figure S1 in supporting information): A solution $\mathrm{A}\left(\mathrm{HAuCl}_{4} .3 \mathrm{H}_{2} \mathrm{O}(1.16 \mathrm{~mL} ; 1 \%[\mathrm{w} / \mathrm{v}])\right.$ and Milli-Q water $(78.84 \mathrm{~mL})$; and a solution $\mathrm{B}$ (sodium citrate $(4 \mathrm{~mL} ; 1.14 \%)$, tannic acid (TA) $(0.025 \mathrm{~mL} ; 1 \%)$ and Milli-Q water $(16 \mathrm{~mL}))$. Solutions A and B were heated to $60^{\circ} \mathrm{C}$ under reflux and stirring then mixed. The molar ratio between gold ions and citrate was [1:6.1]. The mixture was then heated up to $90^{\circ} \mathrm{C}$ for a few minutes to form spherical AuNPs. When the color turned ruby-red, a portion of the sample suspension (portion I) was heated up for 30 min under reflux and stirring to increase the colloidal stability after which the suspension was cooled on ice bath. The other portion (portion II) was used to form gold-silver core-shell NPs and is described later. Finally, gold suspension was kept in amber glassware and stored at $4^{\circ} \mathrm{C}$.

Then, the deposition of Ag shell on AuNPs was realized through reduction of $\mathrm{Ag}^{+}$on AuNPs to obtain Au-Ag core-shell NPs (Au@AgNPs). The molar ratio between $\mathrm{Ag}^{+}$and citrate was also [1:6.1]. The volumes of silver nitrate and sodium citrate stock solutions were determined by estimating a silver shell with uniform thickness of $2 \mathrm{~nm}$ and assuming that the average size of gold core was $13.5 \mathrm{~nm}$. The silver nitrate stock solution $(10 \mathrm{mM})$ was freshly prepared by dissolving $\mathrm{AgNO}_{3} \mathrm{crystals}_{\mathrm{s}}$ in Milli-Q water. A volume of $\mathrm{AgNO}_{3}$ stock solution $(258 \mu \mathrm{L})$ was added to the portion II of AuNPs suspension at $90^{\circ} \mathrm{C}$ after the color change, under reflux and stirring, followed by the rapid addition of the reducing solution (sodium citrate 1\%; $454 \mu \mathrm{L})$. The equations describing the concentration of $(\sim 13.5 \mathrm{~nm})$ AuNPs formed and the volume required to generate a $2 \mathrm{~nm}$ thick Ag shell on such AuNPs are shown in SI (Equation S1 and Equation S2). After addition, suspension was heated up to boiling for $1 \mathrm{~h}$ under reflux and stirring and then cooled on ice bath. Finally, the orange suspension of Au@AgNPs was kept in amber glassware and stored at $4^{\circ} \mathrm{C}$.

Ag-Au core-shell nanoparticle preparation (Ag@AuNPs). The AuNS particles were synthesized via the galvanic replacement method, where the AgNPs served as sacrificial template according to slightly modified procedures. ${ }^{42,48}$ In a typical synthesis, AgNPs were first synthesized by reduction of $\mathrm{AgNO}_{3}$. Sodium citrate solution $\left(1 \mathrm{M}, 50 \mu \mathrm{L}\right.$ ) was added to $\mathrm{AgNO}_{3}$ $(0.2 \mathrm{mM}, 50 \mathrm{~mL})$ at $60^{\circ} \mathrm{C}$. After $5 \mathrm{~min}, \mathrm{NaBH}_{4}(100 \mathrm{mM}, 1 \mathrm{~mL})$ was injected into the solution. The solution was stirred for $2 \mathrm{~h}$ at $60^{\circ} \mathrm{C}$ and allowed to cool down to room temperature. Galvanic replacement of $\mathrm{Ag}$ by Au occurs at room temperature. A $200 \mathrm{mM} \mathrm{NH} 2 \mathrm{OH} . \mathrm{HCl}$ solution $(1 \mathrm{~mL})$ was added to as prepared AgNPs followed by addition of $\mathrm{AgNO}_{3}(0.1 \mathrm{M}, 141 \mu \mathrm{L})$ and citrate $(1 \mathrm{M}, 50 \mu \mathrm{L})$ solutions 5 min later to minimize the formation of AuNPs. ${ }^{57}$ The well stirred solution was kept overnight to allow the reaction to reach completion. $A t 60^{\circ} \mathrm{C}, \mathrm{HAuCl}_{4}(25 \mathrm{mM}, 713 \mu \mathrm{L})$ was added dropwise to the solution under magnetic stirring at $500 \mathrm{rpm}$. The reaction was stopped after $4 \mathrm{~h}$ by lowering the temperature to $25^{\circ} \mathrm{C}$. The final $\mathrm{pH}$ was 4.5 .

An additional silver nitrate solution was added dropwise to a concentration of $0.48 \mathrm{mM}$, which corresponded to the used concentration of AgNPs as template in AuNS synthesis, described previously. This enabled reduction of silver inside gold nanoshell (Ag@AuNPs) and the process was initiated by increasing the pH of purple-colored suspension of AuNS. Thus, after reaching the final concentration, the $\mathrm{pH}$ of the AuNS suspension was adjusted from 4.5 to 8 by adding $\mathrm{NaOH}(0.1 \mathrm{M})$ under magnetic stirring at $500 \mathrm{rpm}$ to afford an orange-colored suspension. Ag@AuNPs suspension was kept in amber glassware and stored at $4^{\circ} \mathrm{C}$.

Engineering of antibody-conjugated nanoparticles. A covalent approach was used to engineer antibody-conjugated NPs via pre-thiolation of antibody $(\mathrm{Ab}) .^{58-59}$ Traut's reagent $(170 \mu \mathrm{M})$ was reacted with $\mathrm{Ab}(0.63 \mathrm{mg} / \mathrm{mL} ; 4.1 \mu \mathrm{M})$ in $40 \mathrm{mM}$ phosphate buffer ( $\mathrm{pH}$ ) at room temperature (RT) to generate sulfhydryl groups. ${ }^{60}$ After $1 \mathrm{~h}$, the thiolated Ab was separated from excess Traut's reagent by purification on Dextran desalting $10 \mathrm{~mL}$ gel filtration column (Hitrap desalt $10 \mathrm{~mL}$, GE Healthcare) using phosphate buffer (pH 7.4) as eluent.

Thiolated $\mathrm{Ab}(70 \mu \mathrm{g} / \mathrm{mL}, 1 \mathrm{~mL})$ was added to Au@AgNPs or Ag@AuNPs suspension (pre-concentrated by seven times, $350 \mathrm{uL})$ and the mixture was incubated for $30 \mathrm{~min}$ at RT. Then, BSA $(0.25 \%$ [w/v]) was added to the bioconjugate suspension to block non-specific binding sites. ${ }^{20,}{ }^{51}$ Finally, the biofunctionalized NPs were concentrated by centrifugation at 10,300 g for 40 min and washed twice to remove excess non-bound Ab or weakly bound Ab. Finally, Ab-NPs were resuspended in $10 \mathrm{mM}$ phosphate buffer (pH 7.4) with BSA $(0.25 \%$ [w/v]).

Antibody quantification by ELISA. After the first centrifugation, the supernatants were collected and Ab concentration was determined by ELISA. SEA $(1 \mu \mathrm{g} / \mathrm{mL}$ in $0.1 \mathrm{M}$ carbonate buffer, $\mathrm{pH} 9.5,100 \mu \mathrm{L})$ was pipetted into each well of a flat-bottomed polystyrene 96-well plate (Greiner bio-one) and incubated overnight at $4^{\circ} \mathrm{C}$. The content was discarded and non-specific sites were blocked by PBS- $0.1 \%$ BSA $(100 \mu \mathrm{L})$ for $1 \mathrm{~h}$ at room temperature. Wells were washed 3 times with PBS-0.05\% Tween $20(150 \mu \mathrm{L})$. Standard solutions of $A b$ or diluted supernatants $(100 \mu \mathrm{L})$ to be quantified were dispensed into the wells. The plate was incubated for $2 \mathrm{~h}$ at room temperature. After washing with PBS-0.05\% Tween $20(3 \times 150 \mu \mathrm{L})$, HRP-labelled goat anti-rabbit IgG conjugate $(1 / 4000,100 \mu \mathrm{L})$ was added to each well and incubated for $1 \mathrm{~h}$ at room temperature. After washing with PBS-0.05\% Tween $20(3 \times 150 \mu \mathrm{L})$, a mixture of $o$-phenylenediamine dihydrochloride (OPD) and $\mathrm{H}_{2} \mathrm{O}_{2}\left(7 \mathrm{mg}\right.$ OPD and $4 \mu \mathrm{L} \mathrm{H} \mathrm{H}_{2}$ in $10 \mathrm{~mL}$ of citrate-phosphate buffer, $\mathrm{pH} 5,100 \mu \mathrm{L}$ ) substrate solution was added to each well. After the color developed, $\mathrm{H}_{2} \mathrm{SO}_{4}(2.5 \mathrm{M}, 50 \mu \mathrm{L})$ was added to each well to stop the enzymatic reaction. After $10 \mathrm{~min}$ in the dark, the absorbance of each well was read at $485 \mathrm{~nm}$ and $415 \mathrm{~nm}$ as reference with a microplate reader (Fluostar Optima, BMG Labtech). The concentration of Ab in diluted supernatants was deduced from the calibration curve established by plotting $\triangle \mathrm{Abs}(\mathrm{Abs} 485-\mathrm{Abs} 415)$ vs $\mathrm{Ab}$ concentration. The resulting value was multiplied by the dilution factor (x200) to determine the actual concentration of $\mathrm{Ab}$ in the supernatants.

SEA immunosensing. Aliquots of SEA solution were serially added to the antibody-conjugated nanoparticles suspension $(1.6 \mathrm{~mL}$ ) in a plastic cuvette (final SEA concentration: 50, 100, 200, 500 and $1000 \mathrm{ng} / \mathrm{mL}$ ). Absorption spectra were 
measured in real time (millisecond temporal resolution and $10^{-2} \mathrm{~nm}$ spectral resolution) using Insplorion XNano II instrument until equilibrium was reached ( $\sim 90 \mathrm{~min})$. The position of the LSPR band was plotted as a function of time using the software provided with the instrument.

UV-Vis spectroscopy. UV-Vis spectra were recorded on a Cary 50 spectrophotometer (Varian, Inc.). Spectral analysis of colloidal suspensions was performed in the range $300-800 \mathrm{~nm}$. Milli- $\mathrm{Q} \mathrm{H}_{2} \mathrm{O}$ was used as the blank. Mathematical determination of $\lambda_{\max }$ was done according to reference. ${ }^{61}$

DLS and zeta potential. Dynamic Light Scattering (DLS), and zeta potential (ELS) measurements were performed using Litesizer $^{\text {TM }} 500$ apparatus (Anton Paar) equipped with a $658 \mathrm{~nm}$ laser operating at $40 \mathrm{~mW}$. The backscattered light collection angle was set at $90^{\circ}$. The zeta potential cuvette has a $\Omega$-shaped capillary tube cuvette with an applied potential of $150 \mathrm{~V}$. The same suspensions were used for DLS and ELS measurements. Each sample was analyzed in triplicate and each measurement was an average of three 30 s runs.

Electron microscopy. Transmission Electron Microscopy (TEM) micrographs of AuNPs, AuNS, Ag@AuNPs and Au@AgNPs were obtained using a JEOL JEM-2100 plus LaB6 (JEOL, Japan) microscope with an acceleration voltage of $200 \mathrm{kV}$ and equipped with an Orius 4 K CDD camera (Gatan, USA). STEM mode coupled with X-ray Energy-Dispersive Spectroscopy (X-EDS) was used to perform elemental mapping of the two metals (lines: Au-L and Ag-L). X-EDS analysis was realized with an Oxford Instrument SDD detector of $80 \mathrm{~mm}^{2}$. X-ray spectra were recorded and processed with Aztec software. The samples were prepared by dropping a dilute suspension onto the carbon-coated copper grids and dried at room temperature before imagining. Size distribution was established by counting a minimum of 400 particles for TEM and was determined using ImageJ Software.

\section{RESULTS AND DISCUSSION}

Nanoparticle synthesis. Two sets of core-shell NPs were prepared: Au@AgNPs and Ag@AuNPs. Prior to synthesis of gold-silver core-shell NPs (Au@AgNPs), pure gold NPs with a core of approx. 14 nm were prepared following the strategy described in Figure 1A.

(A)

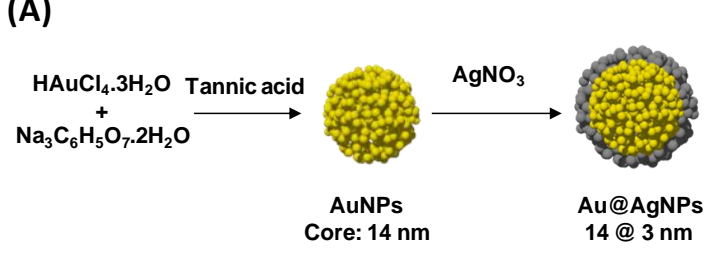

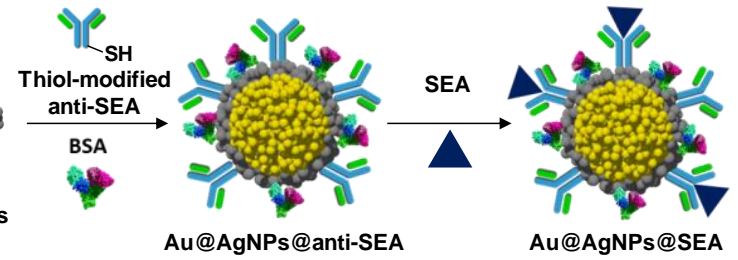

Au@AgNPs@anti-SEA

(B)
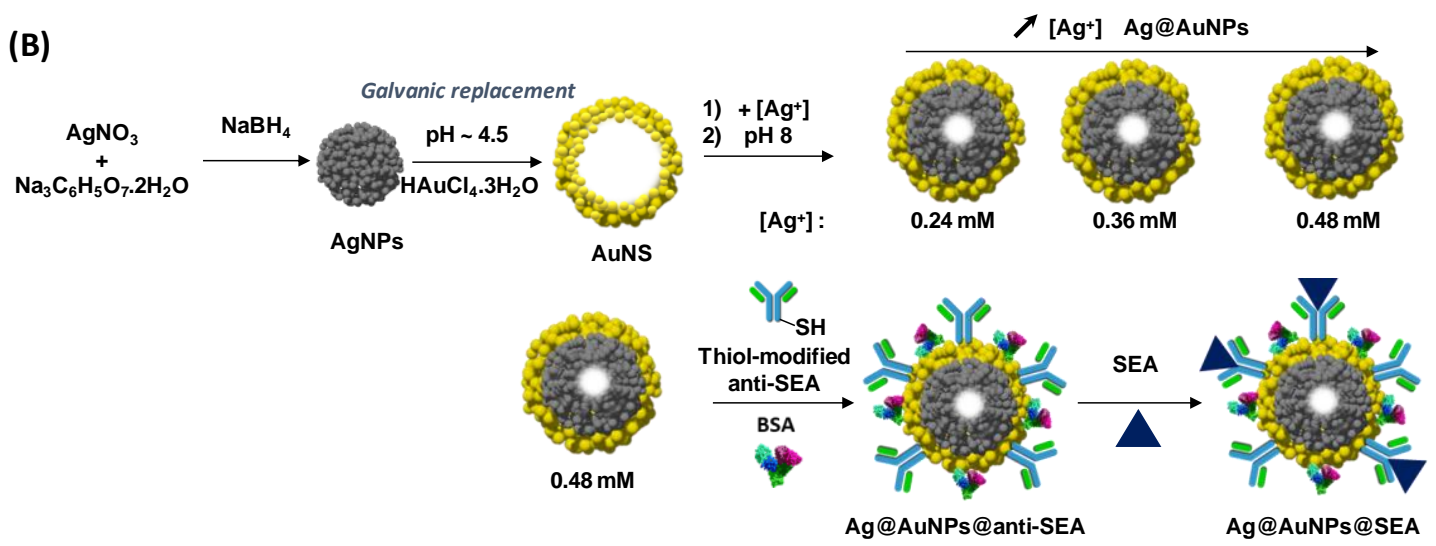

Figure 1. Strategy adopted for the synthesis of core-shell (A) Au@AgNPs and (B) Ag@AuNPs and covalent bioconjugation of anti-SEA antibody as well as the detection of SEA.

Then an Ag shell was grown on the particles to form Au@AgNPs. Silver-gold core-shell NPs (Ag@AuNPs) were synthesized by an original pathway from hollow gold nanoshell (AuNS) by controlling the Ag content in AuNS (Figure 1B). Ag ions up to a concentration of $0.48 \mathrm{mM}$ were added externally to AuNS to yield Ag@AuNPs by pH-triggered reduction (up to 8) in nanoconfinement. ${ }^{37}$ All these colloids were first characterized by UV-Vis spectroscopy, DLS, and zeta potential measurements. The results are displayed in Figure 2 and are summarized in Table 1 (see also supporting information, Figure S2). Then, particles size and distribution were estimated by TEM, see Figure 3. 

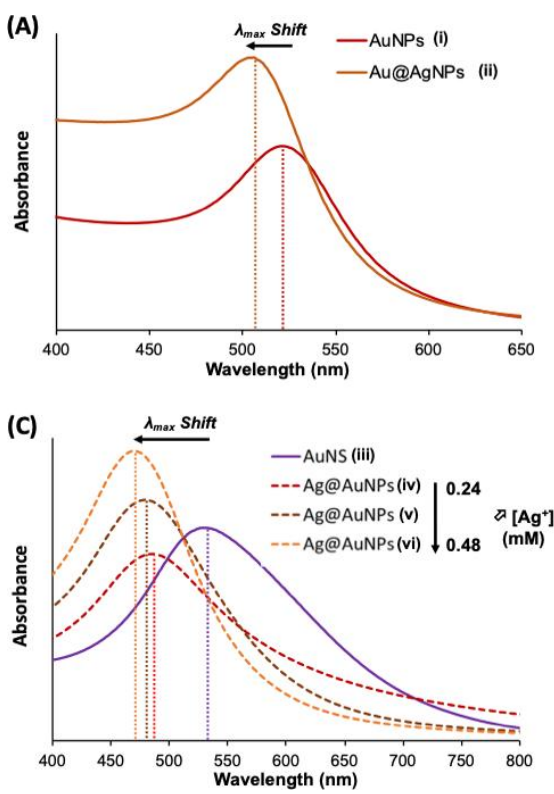

(B)

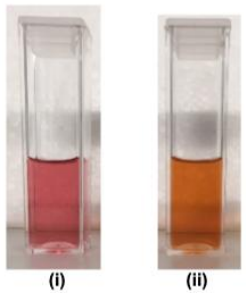

(D)
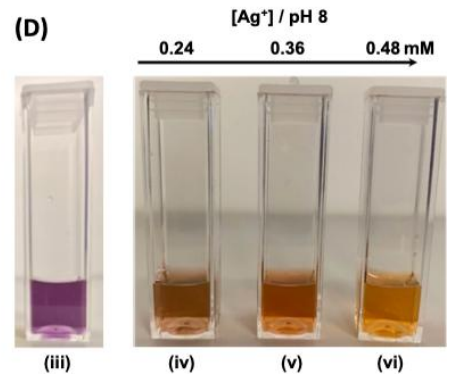

Figure 2. (A) Extinction spectra of (i) pure AuNPs and (ii) core-shell Au@AgNPs; (B) photos of corresponding suspensions. (C) Extinction spectra of (iii) AuNS and Ag@AuNPs, after adding silver nitrate and adjusting the pH from 4.5 to 8: (iv) $\left[\mathrm{Ag}^{+}\right]=0.24 \mathrm{mM},(\mathrm{v})\left[\mathrm{Ag}^{+}\right]=0.36 \mathrm{mM}$ and $(\mathrm{vi})\left[\mathrm{Ag}^{+}\right]=0.48 \mathrm{mM}$; (D) photos of corresponding suspensions.

As shown in Figure 2, the LSPR bands of AuNPs and AuNS are located at $521 \mathrm{~nm}$ and $532 \mathrm{~nm}$ (Table 1), and their color in suspension are red and purple, respectively. The position of the LSPR band of AuNPs is consistent with those previously measured for gold colloids of similar size. ${ }^{20}$ Particle size and distribution were confirmed by TEM observations in Figure 3A-C, with an average size of $14.0 \pm 1.5 \mathrm{~nm}$, for AuNPs, and $31.2 \pm 7.1 \mathrm{~nm}$, for AuNS. Upon silver growth on AuNPs, the LSPR band is blue-shifted to $507 \mathrm{~nm}$ and the color of the core-shell Au@AgNPs colloidal solution turns orange (Figure 2A-B). This color and the position of the LSPR band are consistent with silver growth on the gold core..$^{5,62}$ The same results are observed for Ag incorporation inside AuNS (Figure 2C-D and Table 1). Indeed, the blue-shift of LSPR band increases as the Ag content progressively increases in suspension $(485 \mathrm{~nm}, 479 \mathrm{~nm}$ and $471 \mathrm{~nm}$ for Ag concentration of $0.24,0.36$ and $0.48 \mathrm{mM}$, respectively) and the color also turns to orange/yellow upon Ag addition. Consequently, the LSPR band of Ag@AuNPs can be tuned by controlling the Ag content within AuNS. The average size of Au@AgNPs, deduced from TEM images in Figure 3B, is in good agreement with the growth of a silver shell of $\sim 3 \mathrm{~nm}, 17.2 \pm 2.0 \mathrm{~nm}$. The silver shell is slightly thicker than the targeted value of $2 \mathrm{~nm}$, this is probably due to the higher mean diameter of the original AuNPs (14 vs 13.5 expected). We attempted to grow thicker layers of silver on AuNPs to further blue-shift the position of the LSPR band but this led to the growth of isolated AgNPs (Figure S3). TEM and STEM images (Figure 3C-D and Figure S4) also show that the size of the three different batches of Ag@AuNPs was similar to that of the starting AuNS particles, in agreement with silver growth within the hollow shells. Moreover, traces of residual citrate are visible in the TEM images for AuNS and are consumed during the reduction of silver ions after $\mathrm{AgNO}_{3}$ addition to form $\mathrm{Ag@AuNPs}$ by nanoconfinement effect (compare areas outside the NPs and NP clusters, Figure 3C-D).

(A)

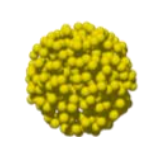

(B)

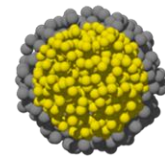

Au@AgNPs

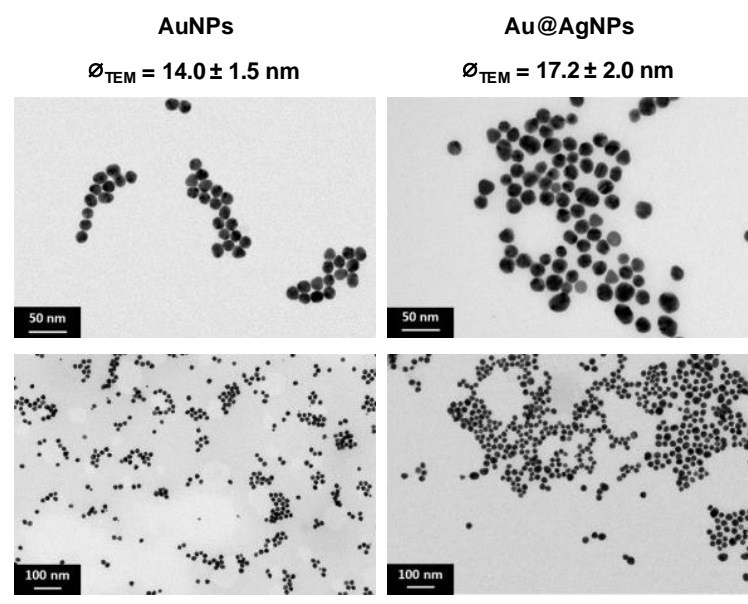

(c)

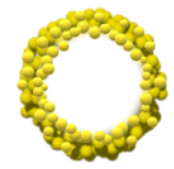

AuNS

$\varnothing_{\text {TEM }}=31.2 \pm 7.1 \mathrm{~nm}$
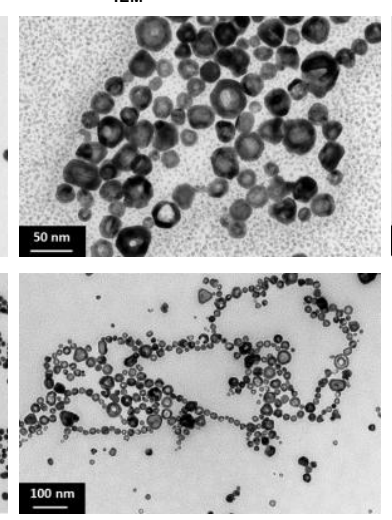

(D)

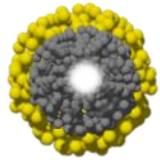

Ag@AuNPs

( $\left.\left[\mathrm{Ag}^{+}\right]=0.48 \mathrm{mM} / \mathrm{pH} 8\right)$

$\varnothing_{\text {TEM }}=31.8 \pm 7.3 \mathrm{~nm}$

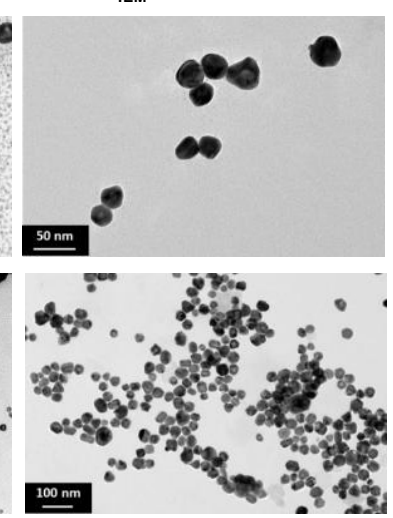


Figure 3. TEM observations of (A) pure AuNPs, (B) core-shell Au@AgNPs, (C) AuNS and (D) core-shell Ag@AuNPs (after adding $\left[\mathrm{Ag}^{+}\right]=0.48 \mathrm{mM}$ in suspension and adjusting the $\mathrm{pH}$ to 8$)$. Their average size was deduced from TEM by counting a minimum of 400 particles.

The hydrodynamic diameter of AuNPs, shown in Table 1, is higher than that obtained from TEM (24.2 nm vs $14.0 \mathrm{~nm}$, respectively), as the measured value includes the surrounding environment. For the core-shell Au@AgNPs, the zeta potential is more negative, approx. $-50 \mathrm{mV}$ than that of AuNPs $(\zeta \approx-40 \mathrm{mV}$ ), a value typically observed for such particles (Figure S2). These observations suggest that silver-coated NPs require more citrates for their stabilization than gold particles. This naturally led to a higher hydrodynamic diameter for these Au@AgNPs, i.e. $\sim 31 \mathrm{~nm}$. The results are very similar between AuNS and different Ag@AuNPs regarding hydrodynamic diameters and zeta potential measurements ( $\sim 52 \mathrm{~nm}$ and $\zeta \approx-39 \mathrm{mV}$, respectively) (Table 1 and Figure S2). However, a slight decrease in zeta potential is observed for Ag@AuNPs $\left(\left[\mathrm{Ag}^{+}\right]=0.24 \mathrm{mM}\right)$ resulting in an increase of the hydrodynamic diameter and resulting in less stable nanoparticles. Indeed, AuNS lose their colloidal stability by removing excess citrate in suspension. Progressive filling of the inner cavity of AuNS with Ag occurs until a solid core of Ag is formed and Ag@AuNPs remain stable in suspension. Consequently, we focused on Ag@AuNPs $\left(\left[\mathrm{Ag}^{+}\right]=0.48 \mathrm{mM}\right)$ and Au@AgNPs in order to compare their potential as well as their efficacy for SEA immunosensing after engineering the antibody-conjugated core-shell NPs and deploying them for naked-eye biosensing.

To ensure their successful synthesis, Au@AgNPs, AuNS and Ag@AuNPs were further characterized using HR-TEM and X-EDS. The resulting STEM elemental mapping ( $\mathrm{Ag}$, Au and overlay) and elemental profile are shown in Figure 4. These observations highlighted the silver growth on AuNPs to obtain Au@AgNPs (Figure 4A) and the pH-triggered silver reduction to completely fill the inner volume for Ag@AuNPs (Figure 4B-C and Figure S4). X-EDS data show that the grouped $\mathrm{Au} @ A g N P s\left(\approx \mathrm{Au}_{69} \mathrm{Ag}_{31}\right)$ (sample ii, Table 1) matched perfectly the theoretical atomic percentages of gold and silver in $\mathrm{Au} @ A g N P s$, i.e. $\approx \mathrm{Au}_{66} \mathrm{Ag}_{34}$. However, when looking at individual Au@AgNPs, the amount of silver is slightly lower with an average composition $\approx \mathrm{Au}_{79} \mathrm{Ag}_{21}$. This small difference may have two origins. First, some small silver nanoparticles, barely detectable on the TEM images and in the LSPR bands may be formed. This first assumption is consolidated by additional experiments we ran to increase the shell thickness in Au@AgNPs ( $>3$ nm, not shown) that led to formation separate AgNPs in the colloidal suspension. The second assumption would be that not all the silver introduced to the solution is reduced (note X-EDS analysis shows the element regardless of its oxidation state). In addition, elemental mapping (Ag, Au and overlay in Figure 4A) clearly shows a relatively homogeneous layer of silver on gold core. Regarding Ag@AuNPs, X-EDS data confirms the STEM findings with an incremental increase in Ag content in AuNS (Table 1 and Figure S4). Therefore, we conclude that the synthesis of Au@AgNPs and Ag@AuNPs was successful.

(A)

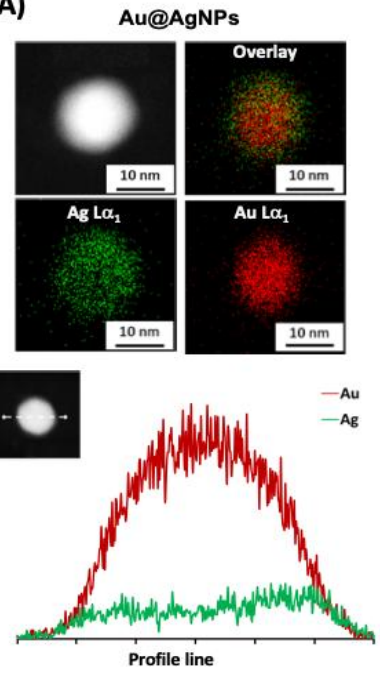

(B)

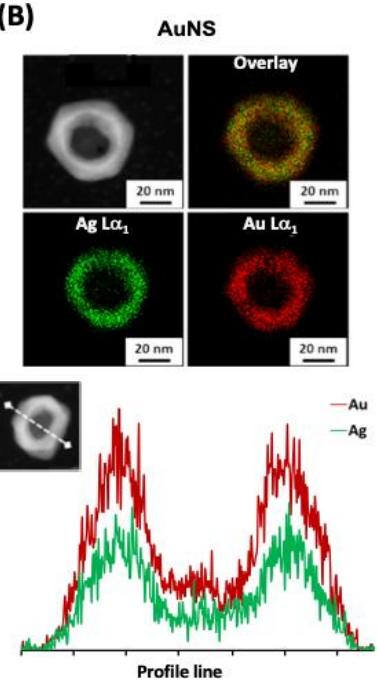

(C) Ag@AuNPs

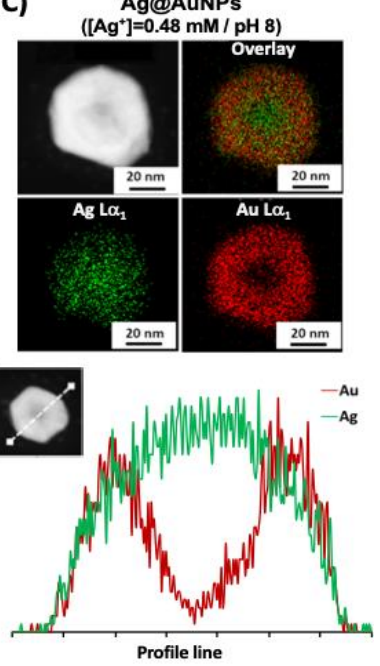

Figure 4. STEM elemental mapping (Ag, Au and overlay) of (A) Au@AgNPs, (B) AuNS and (C) Ag@AuNPs $\left(\left[\mathrm{Ag}^{+}\right]=0.48 \mathrm{mM}\right)$ and elemental profiles along the white hatched line.

Bioconjugate engineering. Ab-NPs bioconjugates were engineered by attaching anti-SEA antibody to Au@AgNPs or Ag@AuNPs following the procedure schematized in Figure 1. Prior to grafting on the surfaces, Ab was derivatized by Traut's reagent to generate thiol groups allowing its covalent anchoring to the metallic nanoparticle surface by displacing the capping citrate ligands. The increase of particles size due to the grafted proteins was evidenced by DLS measurements as the hydrodynamic diameter of Ab-Au@AgNPs and Ab-Ag@AuNPs increased to 180.6 nm and 182 nm, respectively (Table S1). The zeta potential of these core-shell NPs in phosphate buffer (10 mM; pH 7.4$)$ also increased from -50.5 to -18.8 mV for Ab-Au@AgNPs and from -38.9 to -15.6 mV for Ab-Ag@AuNPs (Table S1) in agreement with previously reported data. ${ }^{20,51}$ These successive steps were monitored by UV-Vis spectroscopy as shown in Figure 5. The position of the LSPR band $\left(\lambda_{\max }\right.$ shift) was determined by calculating the first derivative of each spectrum. ${ }^{8,61}$ 
Table 1. Summary of physical properties of (i) AuNPs, (ii) Au@AgNPs, (iii) AuNS and Ag@AuNPs with various addition content of Ag inside AuNS ((iv) $\left.\left[\mathrm{Ag}^{+}\right]=0.24 \mathrm{mM},(\mathrm{v})\left[\mathrm{Ag}^{+}\right]=0.36 \mathrm{mM},(\mathrm{vi})\left[\mathrm{Ag}^{+}\right]=0.48 \mathrm{mM}\right)$.

\begin{tabular}{|c|c|c|c|c|}
\hline Samples & $\begin{array}{l}\lambda_{\max } \\
(\mathrm{nm})\end{array}$ & $\begin{array}{l}\text { DLS } \\
(\mathrm{nm})\end{array}$ & $\begin{array}{c}\text { 万-potential } \\
(\mathrm{mV})\end{array}$ & X-EDS (at. \%) \\
\hline $\mathrm{i}$ & 521 & 24.2 & -42.3 & $\mathrm{Au}_{100}$ \\
\hline ii & 507 & 31.0 & -50.5 & $\mathrm{Au}_{69} \mathrm{Ag}_{31} / / \mathrm{Au}_{79} \mathrm{Ag}_{21}$ \\
\hline iii & 532 & 50.9 & -39.5 & $\mathrm{Ag}_{60} \mathrm{Au}_{40}$ \\
\hline iv & 485 & 61.2 & -34.1 & $\mathrm{Ag}_{67} \mathrm{Au}_{33}$ \\
\hline $\mathrm{v}$ & 479 & 52.1 & -39 & $\mathrm{Ag}_{70} \mathrm{Au}_{30}$ \\
\hline vi & 471 & 54.3 & -38.9 & $\mathrm{Ag}_{72} \mathrm{Au}_{28}$ \\
\hline
\end{tabular}

The first observation is that under identical conditions, the LSPR band red-shift was more pronounced for the core-shell Ag@AuNPs compared to Au@AgNPs, 23 nm vs $17.8 \mathrm{~nm}$, respectively (Figure 5). This corroborated the assumption that the RI sensitivity is improved with increasing Ag amount, as confirmed by X-EDS data (Table 1). ${ }^{5,63}$ Besides, a third batch of colloids, i.e. pure AuNPs of $17 \mathrm{~nm}$ in diameter, was prepared to have particles with similar sizes to Au@AgNPs for comparison of the efficiency of Ab chemisorption. The LSPR-shift for this AuNP is reduced by a factor two yielding a red-shift of $8 \mathrm{~nm}$ (Figure S5). In addition, and more importantly, the attachment of Ab to Au@AgNPs was readily observable by naked-eye as the color switched from orange to red (see Figure 5A(ii)), while AuNPs and Ab-AuNPs were both reddish (Figure S5). The color change for Ag@AuNPs is less obvious as the suspension changed from orange to deep orange/brownish (Figure 5B(ii)). Unfortunately, the LSPR signature of the resulting Ab-Au@AgNPs bioconjugates was no longer located in the desired spectral window, which compromises the visualization of SEA binding. Nevertheless, we pursued the immunosensing to see whether the better RI sensitivity of both core-shell colloids may enhance their response to SEA.
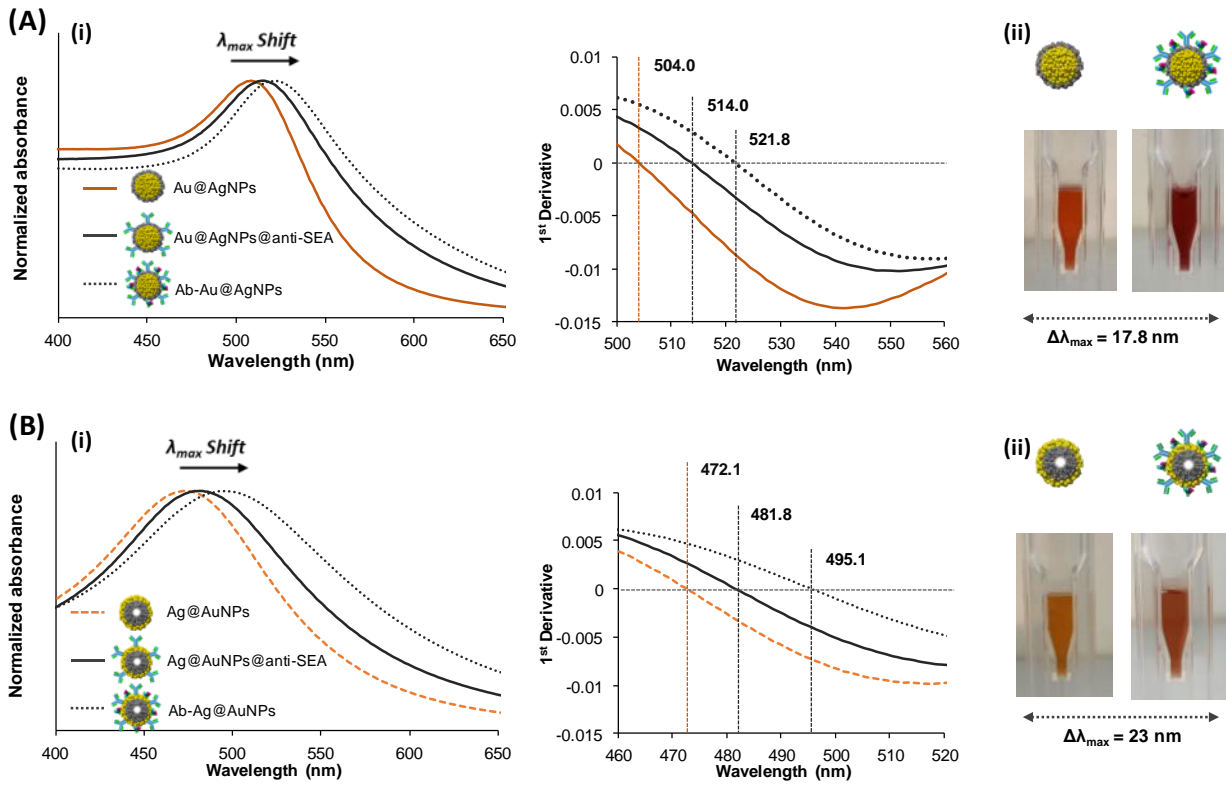

(ii)

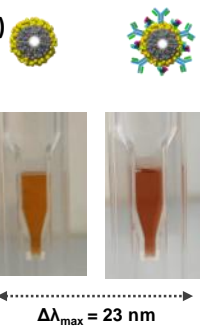

Figure 5. (A) (i) UV-Visible spectra and their first derivative recorded upon Ab conjugation to Au@AgNPs; (ii) photos of the suspensions before (left) and after (right) bioconjugation. (B) (i) UV-Visible spectra and their first derivative recorded upon Ab bioconjugation to Ag@AuNPs; (ii) photos of the suspensions before (left) and after (right) bioconjugation.

Prior to SEA immunosensing, the surface coverage of antibodies for each bioconjugate was indirectly estimated by measuring the unbound antibody quantity remaining in the supernatant using enzyme-linked immunosorbent assay (ELISA). This information is very important when comparing the efficiency of the bioconjugates for biosensing as it can be directly related to the amount and accessibility of the recognition sites. ELISA gave the actual concentrations of Ab in the supernatants i.e. $14.8 \mu \mathrm{g} / \mathrm{mL}$ for Au@AgNPs and 19.4 $\mu \mathrm{g} / \mathrm{mL}$ for Ag@AuNPs (Figure S6). Surprisingly, these unbound Ab concentrations indicate a higher grafting of Ab on Au@AgNPs compared to Ag@AuNPs despite a lower shift of the LSPR band and smaller particle size. The reactivity of thiol groups towards silver and gold is also supposed to be similar, yet, we can expect slight differences, which may influence the coverage on the NPs. As stated above, the amount of citrate needed to stabilize Au@AgNPs is higher than that required for Ag@AuNPs. This may be at the origin of this difference in coverages. It is also important to note that ELISA was performed on the supernatants. This method has been shown to overestimate the amount of adsorbed Ab. 64

SEA immunosensing. In this experiment, the position of the LSPR bands of the colloidal suspensions of Ab-Au@AgNPs and Ab-Ag@AuNPs was monitored over time upon addition of increasing amounts of SEA in the range 0 to $1000 \mathrm{ng} / \mathrm{mL}$ (Figure 6A-C). A progressive shift of the LSPR band was observed and a dose-response curve was established by plotting 
the band shift $\Delta \lambda$ measured after stabilization versus the concentration of analyte (Figure 6B-D). The detailed fit parameters showing the accuracy of the measurement $\left(\chi^{2}\right.$ and $\mathrm{R}^{2}$ values) are summarized in Table S2. Following the red-shift of LSPR bands to assess SEA recognition led to a large enhancement of the dynamic range compared to our previously published data. ${ }^{20}$ Yet, at this step, no remarkable differences are observed between the responses of Au@AgNPs and Ag@AuNPs immunoprobes towards SEA. Indeed, addition of SEA led to red-shift of the LSPR band which reached a total shift of $\Delta \lambda=6.3 \mathrm{~nm}$ and $\Delta \lambda=6.6 \mathrm{~nm}$ at saturation for Au@AgNPs@SEA and Ag@AuNPs@SEA, respectively, upon exposure to $500 \mathrm{ng} / \mathrm{mL}$ SEA (Figure 6); no further shift is observed for higher SEA concentrations. Through the Langmuir fit on adsorption isotherm curves, both Ab-Au@AgNPs and Ab-Ag@AuNPs show a high affinity between anti-SEA and SEA while Ab-Ag@AuNPs exhibited a better affinity (Figure 6B-D). The dissociation constants for Au@AgNPs and Ag@AuNPs are $168.8 \pm 35.3 \mathrm{ng} / \mathrm{mL}(6.0 \pm 1.3 \mathrm{nM})$ and $134.6 \pm 27.1 \mathrm{ng} / \mathrm{mL}(4.8 \pm 1.0 \mathrm{nM})$, respectively and the limits of detection (LOD) of SEA are estimated to equal $5.4 \mathrm{ng} / \mathrm{mL}(0.2 \mathrm{nM})$ and $10.6 \mathrm{ng} / \mathrm{mL}(0.4 \mathrm{nM})$, respectively. These LOD results points toward a high analytical performance for both systems and are of the same orders of magnitude as for the Ab-AuNPs bioconjugate, reported in a previous work. ${ }^{20}$
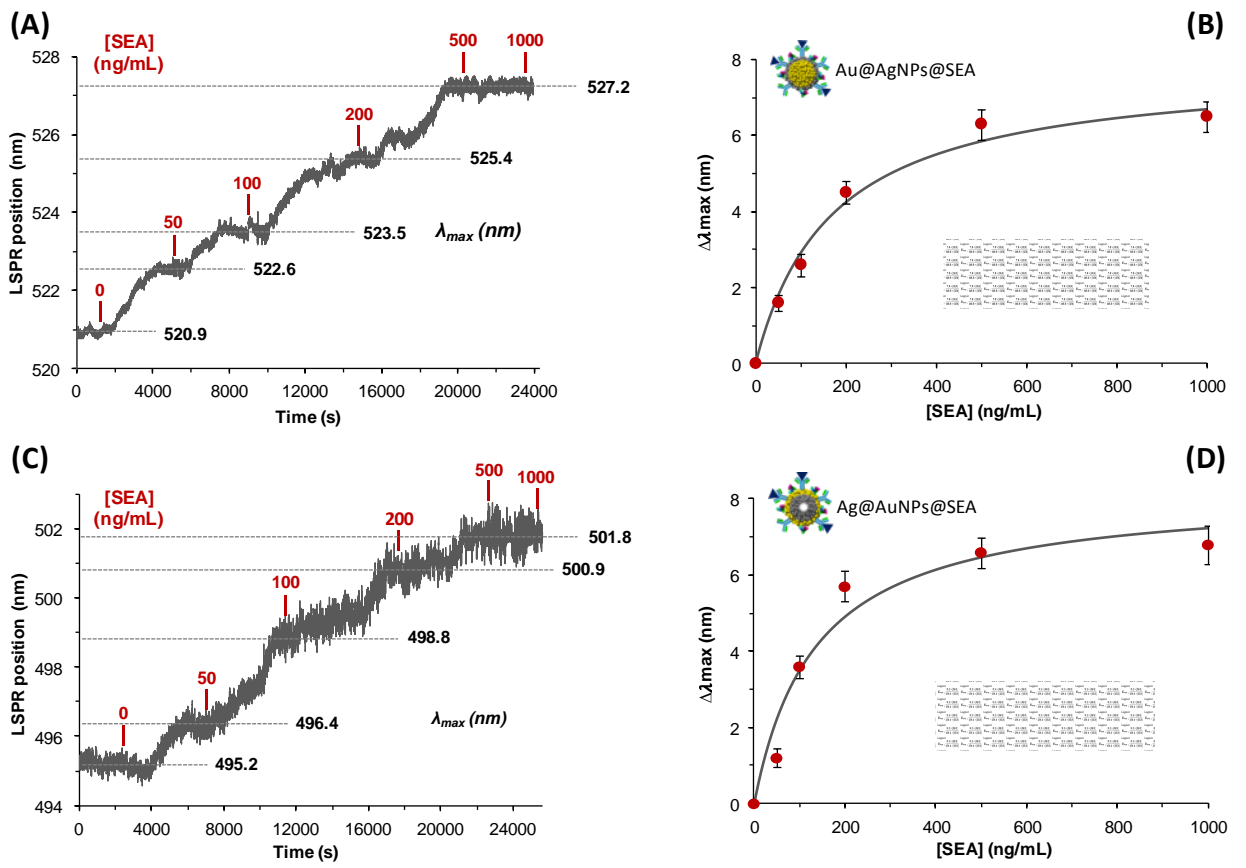

Figure 6. (A) LSPR band position vs time and (B) dose-response curve established by plotting $\Delta \lambda$ at stabilization $v s$ [SEA] for Ab-Au@AgNPs immunoprobe. (C) LSPR band position vs time and (D) dose-response curve established by plotting $\Delta \lambda$ at stabilization vs [SEA] for Ab-Ag@AuNPs immunoprobe. Langmuir fit was applied and the equation was added to the figure.

In addition, we were able to visualize the optical response of the immunosensors to SEA by naked-eye readout even for relatively small red-shifts $(<7 \mathrm{~nm}$ ) (see Figure 6). We observed a significant color change from orange to red for Ab-Ag@AuNPs suspension after addition of $200 \mathrm{ng} / \mathrm{mL}$ SEA, and remains unchanged when adding up to $500 \mathrm{ng} / \mathrm{mL}$ SEA as shown in Figure 7A. This confirms the predictions stated above that the LSPR band of the Ab-Ag@AuNPs was shifted in the optimal window $(\sim 500 \mathrm{~nm})$ to yield an orange to red color change upon for naked-eye interrogation. ${ }^{45}$ In the case of plasmonic Au@AgNPs, the LSPR band, after Ab grafting, was already outside the window targeted in this work. Nevertheless, SEA could be visually detected even if the color change was less evident: from red without SEA to pinkish tinge with SEA (Figure 7B). Thus, the elaboration of plasmonic Ag-Au core shell NPs, with well-controlled parameters (Ag@AuNPs), provide significantly better results for naked-eye toxin biosensing. 


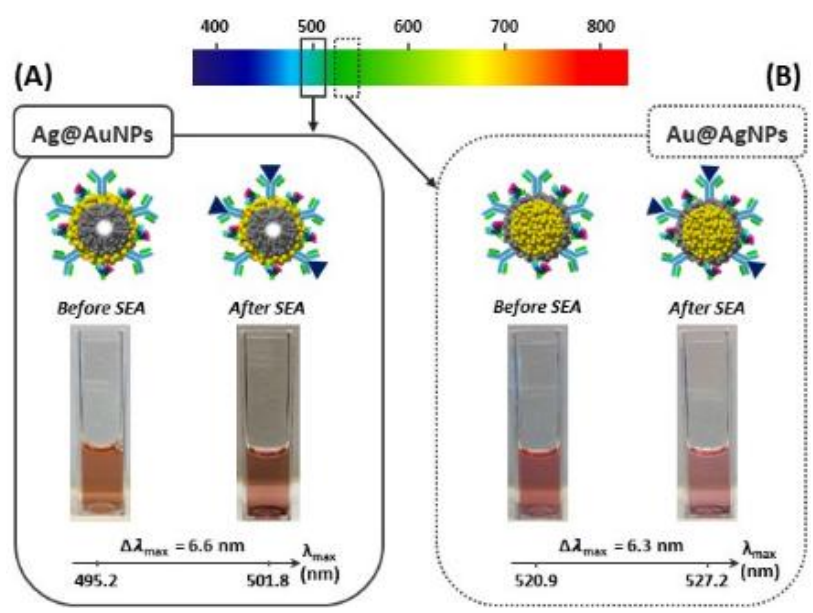

Figure 7. Photos of the suspensions, before and after adding SEA to Ag Au core-shell NP suspensions: the color change is visually detected from (A) orange without SEA to red with SEA for Ag@AuNPs and (B) from red without SEA to pinkish tinge with SEA for Au@AgNPs. Photos were taken after the last addition of SEA (500 ng/mL) due to the limit of in-situ device.

\section{CONCLUSIONS}

$\mathrm{Au}-\mathrm{Ag}$ core-shell NPs were engineered by growing a thin layer of Ag on citrate-capped AuNPs, while Ag-Au core-shell NPs were obtained by controlling the Ag reduction and deposition inside hollow gold nanoshells by nanoconfinement effect. Two immunoprobes (Ab-Au@AgNPs and Ab-Ag@AuNPs) were engineered by conjugating thiolated anti-SEA antibody to Au@AgNPs and Ag@AuNPs. These immunoprobes were applied to detect and quantify SEA by LSPR in real time using a UVVis spectrometer. The one step LSPR assays of SEA with Ab Au@AgNPs and Ab Ag@AuNPs bioconjugates afforded remarkable analytical performances at the $\mathrm{ng} / \mathrm{mL}$ level and LOD were estimated spectroscopically to be $5.4 \mathrm{ng} / \mathrm{mL}(0.2 \mathrm{nM})$ and $10.6 \mathrm{ng} / \mathrm{mL}(0.4 \mathrm{nM})$, respectively. The benefit gained by combining Au and Ag noble metals in core-shell entities was twofold: (1) the RI sensitivity of the NPs was shown to be higher, and (2) the wavelength window allowed visual detection of biomolecules binding by tuning the LSPR band of Au@AgNPs and Ag@AuNPs. Indeed, starting from the antibody conjugation step, the LSPR band of both core-shell NPs were markedly red-shifted. This led to the color change of the colloidal suspension, initially orange, that visually turned to red for Au@AgNPs and deep orange for Ag@AuNPs. Thus, grafting of Ab could be visualized by naked-eye. Yet, the extent of this red-shift moved the LSPR band of Au@AgNPs outside the ideal spectral window targeted in this work. Therefore, color change detectable by naked-eye was less obvious upon subsequent binding of SEA. In contrast, the LSPR band of Ab-Ag@AuNPs immunoprobe was still within the desired spectral window and the color of suspension changed from orange to red upon binding of SEA, even for similar red-shift of LSPR band. The one-step LSPR assays of SEA with Ab-Au@AgNPs and Ab-Ag@AuNPs bioconjugates afforded remarkable analytical performances at the $\mathrm{ng} / \mathrm{mL}$ level and LOD were estimated to be $5.4 \mathrm{ng} / \mathrm{mL}(0.2 \mathrm{nM})$ and $10.6 \mathrm{ng} / \mathrm{mL}(0.4 \mathrm{nM})$, respectively. Finally, gold and silver core-shell NPs, and more particularly Ag@AuNPs synthesized from hollow gold nanoshells, appear to be an innovative tool in medical diagnostic and environmental monitoring, especially in situations where basic laboratory infrastructure is sparse or non-existent. Our ongoing work is to improve the specificity of these plasmonic nanoparticles in complex matrices as milk and then, expand the potential of these biosensors to other toxins/biomolecules.

\section{ASSOCIATED CONTENT}

\section{Supporting Information}

Effect of the TA concentration in the reducing solution on AuNPs size; Calculation of AuNPs concentration; Calculation to determine the added Ag content for dedicated shell thickness on AuNPs; Dynamic light scattering (DLS), and zeta potential of pure AuNPs, core-shell Au@AgNPs, AuNS and Ag@AuNPs after adding silver nitrate in suspension and adjusting the pH to 8; Additional growth of silver shell on gold nanoparticles; STEM/X-EDS elemental of AuNS and Ag@AuNPs according to silver addition in suspension at pH 8; Summary of physical properties of Au@AgNPs, Ab-Au@AgNPs, Ag@AuNPs and Ab-Ag@AuNPs; UV-Visible spectra and their first derivative during anti-SEA antibody conjugation to $17 \mathrm{~nm}$-diameter AuNPs; Standard curve for quantifying anti-SEA in the supernatant by ELISA; Fit parameters for SEA immunosensing.

\section{AUTHOR INFORMATION}

\section{Corresponding Authors}

*E-mail: souhir.boujday@sorbonne-universite.fr (S.B.).

*E-mail: bliedberg@ntu.edu.sg (B.L.).

\section{ORCID}


Alexis Loiseau: 0000-0003-0881-5637

Lu Zhang: 0000-0002-2757-4173

Michèle Salmain: 0000-0003-3039-5659

Bo Liedberg: 0003-0003-2883-6953

Souhir Boujday: 0000-0002-5500-0951

\section{Author Contributions}

A.L. and L.Z. contributed equally. The manuscript was written through contributions of all authors. All authors have given approval to the final version of the manuscript.

Notes

The authors declare no competing financial interest

\section{ACKNOWLEDGMENT}

We would like to thank the French-Singaporean PHC Merlion program (grant 5.03.15) for financial support. B.L. also acknowledge support from the Institute for Nanomedicine jointly established between Northwestern University and Nanyang Technological University. S.B., A.L., and M.S. acknowledge the ANR-FWF grant "NanoBioSensor", ANR-15- CE29-0026-02.

\section{REFERENCES}

1. $\quad$ Elghanian, R.; Storhoff, J. J.; Mucic, R. C.; Letsinger, R. L.; Mirkin, C. A., Selective Colorimetric Detection of Polynucleotides Based on the Distance-Dependent Optical Properties of Gold Nanoparticles. Science 1997, 277 (5329), 1078-1081.

2. Daniel, M.-C.; Astruc, D., Gold Nanoparticles: Assembly, Supramolecular Chemistry, Quantum-Size-Related Properties, and Applications Toward Biology, Catalysis, and Nanotechnology. Chem. Rev. 2004, 104 (1), 293-346.

3. Anker, J. N.; Hall, W. P.; Lyandres, O.; Shah, N. C.; Zhao, J.; Van Duyne, R. P., Biosensing with Plasmonic Nanosensors. Nat. Mater. 2008, 7, 442-453.

4. Hui, S., Plasmonic Nanoparticles: Towards the Fabrication of Biosensors. In IOP Conf. Ser.: Mater. Sci. Eng., 2015; Vol. 87, p 012009.

5. Loiseau, A.; Asila, V.; Boitel-Aullen, G.; Lam, M.; Salmain, M.; Boujday, S., Silver-Based Plasmonic Nanoparticles for and Their Use in Biosensing. Biosensors 2019, 9 (2), 78-116.

6. Gopinath, S. C. B., Biosensing Applications of Surface Plasmon Resonance-Based Biacore Technology. Sens. Actuators, B 2010, $150(2), 722-733$.

7. $\quad$ Mayer, K. M.; Hafner, J. H., Localized Surface Plasmon Resonance Sensors. Chem. Rev. 2011, 111 (6), $3828-3857$.

8. $\quad$ Chen, P.; Tran, N. T.; Wen, X.; Xiong, Q.; Liedberg, B., Inflection Point of the Localized Surface Plasmon Resonance Peak: A General Method to Improve the Sensitivity. ACS Sens. 2017, 2 (2), 235-242.

9. Mak, W. C.; Beni, V.; Turner, A. P. F., Lateral-Flow Technology: from Visual to Instrumental. TrAC, Trends Anal. Chem. 2016, 79, 297-305.

10. Zhao, W.; Brook, M. A.; Li, Y. F., Design of Gold Nanoparticle-Based Colorimetric Biosensing Assays. ChemBioChem 2008, 9 (15), 2363-2371.

11. Zhang, Z.; Wang, H.; Chen, Z.; Wang, X.; Choo, J.; Chen, L., Plasmonic Colorimetric Sensors Based on Etching and Growth of Noble Metal Nanoparticles: Strategies and Applications. Biosens. Bioelectron. 2018, 114, 52-65.

12. Lee, K.-S.; El-Sayed, M. A., Gold and Silver Nanoparticles in Sensing and Imaging: Sensitivity of Plasmon Response to Size, Shape, and Metal Composition. J. Phys. Chem. B 2006, 110 (39), 19220-19225.

13. Rycenga, M.; Cobley, C. M.; Zeng, J.; Li, W.; Moran, C. H.; Zhang, Q.; Qin, D.; Xia, Y., Controlling the Synthesis and Assembly of Silver Nanostructures for Plasmonic Applications. Chem. Rev. 2011, 111 (6), 3669-3712.

14. Englebienne, P., Use of Colloidal Gold Surface Plasmon Resonance Peak Shift to Infer Affinity Constants from the Interactions Between Protein Antigens and Antibodies Specific for Single or Multiple Epitopes. Analyst 1998, 123 (7), $1599-1603$.

15. Mortazavi, D.; Kouzani, A. Z.; Kaynak, A.; Duan, W., Nano-Plasmonic Biosensors: A Review. In The 2011 IEEE/ICME International Conference on Complex Medical Engineering, 2011; pp 31-36.

16. Sepúlveda, B.; Angelomé, P. C.; Lechuga, L. M.; Liz-Marzán, L. M., LSPR-Based Nanobiosensors. Nano Today 2009, 4 (3), 244-251.

17. Chen, P.; Liu, X.; Goyal, G.; Tran, N. T.; Shing Ho, J. C.; Wang, Y.; Aili, D.; Liedberg, B., Nanoplasmonic Sensing from the Human Vision Perspective. Anal. Chem. 2018, 90 (7), 4916-4924.

18. Steinbrück, A.; Stranik, O.; Csaki, A.; Fritzsche, W., Sensoric Potential of Gold-Silver Core-Shell Nanoparticles. Anal. Bioanal. Chem. 2011, 401 (4), 1241-1249.

19. Lu, L.; Burkey, G.; Halaciuga, I.; Goia, D. V., Core-Shell Gold/Silver Nanoparticles: Synthesis and Optical Properties. J. Colloid Interface Sci. 2013, 392, 90-95.

20. Ben Haddada, M.; Hu, D.; Salmain, M.; Zhang, L.; Peng, C.; Wang, Y.; Liedberg, B.; Boujday, S., Gold Nanoparticle-Based Localized Surface Plasmon Immunosensor for Staphylococcal Enterotoxin A (SEA) Detection. Anal. Bioanal. Chem. 2017, 409 (26), 6227-6234.

21. Liu, S.; Chen, G.; Prasad, P. N.; Swihart, M. T., Synthesis of Monodisperse Au, Ag, and Au-Ag Alloy Nanoparticles with Tunable Size and Surface Plasmon Resonance Frequency. Chem. Mater. 2011, 23 (18), 4098-4101.

22. Noguez, C., Surface Plasmons on Metal Nanoparticles: the Influence of Shape and Physical Environment. J. Phys. Chem. C 2007, 111 (10), 3806-3819.

23. Lee, K.-C.; Lin, S.-J.; Lin, C.-H.; Tsai, C.-S.; Lu, Y.-J., Size Effect of Ag Nanoparticles on Surface Plasmon Resonance. Surf. Coat. Technol. 2008, 202 (22-23), 5339-5342.

24. Lismont, M.; Dreesen, L., Comparative Study of Ag and Au Nanoparticles Biosensors Based on Surface Plasmon Resonance Phenomenon. Mater. Sci. Eng. C 2012, 32 (6), 1437-1442. 
25. Boisselier, E.; Astruc, D., Gold Nanoparticles in Nanomedicine: Preparations, Imaging, Diagnostics, Therapies and Toxicity. Chem. Soc. Rev. 2009, 38 (6), 1759-1782.

26. Dreaden, E. C.; Alkilany, A. M.; Huang, X.; Murphy, C. J.; El-Sayed, M. A., The Golden Age: Gold Nanoparticles for Biomedicine. Chem. Soc. Rev. 2012, 41 (7), 2740-2779.

27. Jakab, A.; Rosman, C.; Khalavka, Y.; Becker, J.; Trügler, A.; Hohenester, U.; Sönnichsen, C., Highly Sensitive Plasmonic Silver Nanorods. ACS Nano 2011, 5 (9), 6880-6885.

28. Navas, M. P.; Soni, R. K., Laser-Generated Bimetallic Ag-Au and Ag-Cu Core-Shell Nanoparticles for Refractive Index Sensing. Plasmonics 2015, 10 (3), 681-690.

29. Liz-Marzan, L. M., Tailoring Surface Plasmons Through the Morphology and Assembly of Metal Nanoparticles. Langmuir 2006, 22 (1), 32-41.

30. Dong, P.; Wu, Y.; Guo, W.; Di, J., Plasmonic Biosensor Based on Triangular Au/Ag and Au/Ag/Au Core/Shell Nanoprisms onto Indium Tin Oxide Glass. Plasmonics 2013, 8 (4), 1577-1583.

31. Sun, L.; Li, Q.; Tang, W.; Di, J.; Wu, Y., The Use of Gold-Silver Core-Shell Nanorods Self-Assembled on a Glass Substrate Can Substantially Improve the Performance of Plasmonic Affinity Biosensors. Microchim. Acta 2014, 181 (15), $1991-1997$.

32. Hao, J.; Xiong, B.; Cheng, X.; He, Y.; Yeung, E. S., High-Throughput Sulfide Sensing with Colorimetric Analysis of Single AuAg Core-Shell Nanoparticles. Anal. Chem. 2014, 86 (10), 4663-4667.

33. Yan, W.; Yang, L.; Zhuang, H.; Wu, H.; Zhang, J., Engineered "Hot" Core-Shell Nanostructures for Patterned Detection of Chloramphenicol. Biosens. Bioelectron. 2016, 78, 67-72.

34. Guo, Y.; Wu, J.; Li, J.; Ju, H., A Plasmonic Colorimetric Strategy for Biosensing through Enzyme Guided Growth of Silver Nanoparticles on Gold Nanostars. Biosens. Bioelectron. 2016, 78, 267-273.

35. Mao, K.; Yang, Z.; Li, J.; Zhou, X.; Li, X.; Hu, J., A Novel Colorimetric Biosensor Based on Non-Aggregated Au@Ag Core-Shell Nanoparticles for Methamphetamine and Cocaine Detection. Talanta 2017, 175, 338-346.

36. Cortie, M. B.; McDonagh, A. M., Synthesis and Optical Properties of Hybrid and Alloy Plasmonic Nanoparticles. Chem. Rev. 2011, $111(6), 3713-3735$

37. Ferrando, R.; Jellinek, J.; Johnston, R. L., Nanoalloys: from Theory to Applications of Alloy Clusters and Nanoparticles. Chem. Rev. 2008, 108 (3), 845-910.

38. Morriss, R. H.; Collins, L. F., Optical Properties of Multilayer Colloids. J. Chem. Phys. 1964, 41 (11), $3357-3363$.

39. Yang, X.; Wang, Y.; Liu, Y.; Jiang, X., A Sensitive Hydrogen Peroxide and Glucose Biosensor Based on Gold/Silver Core-Shell Nanorods. Electrochim. Acta 2013, 108, 39-44.

40. Lu, L.; Wang, H.; Zhou, Y.; Xi, S.; Zhang, H.; Hu, J.; Zhao, B., Seed-Mediated Growth of Large, Monodisperse Core-Shell GoldSilver Nanoparticles with Ag-like Optical Properties. Chem. Commun. 2002, (2), 144-145.

41. Halas, N., Playing with Plasmons: Tuning the Optical Resonant Properties of Metallic Nanoshells. MRS Bull. 2005, 30 (5), 362-367.

42. Prevo, B. G.; Esakoff, S. A.; Mikhailovsky, A.; Zasadzinski, J. A., Scalable Routes to Gold Nanoshells with Tunable Sizes and Response to Near-Infrared Pulsed-Laser Irradiation. Small 2008, 4 (8), 1183-1195.

43. Russo, L.; Merkoçi, F.; Patarroyo, J.; Piella, J.; Merkoçi, A.; Bastús, N. G.; Puntes, V., Time- and Size-Resolved Plasmonic Evolution with nm Resolution of Galvanic Replacement Reaction in AuAg Nanoshells Synthesis. Chem. Mater. 2018, 30 (15), 50985107.

44. Goodman, A. M.; Cao, Y.; Urban, C.; Neumann, O.; Ayala-Orozco, C.; Knight, M. W.; Joshi, A.; Nordlander, P.; Halas, N. J., The Surprising in Vivo Instability of Near-IR-Absorbing Hollow Au-Ag Nanoshells. ACS Nano 2014, 8 (4), 3222-3231.

45. Li, Y.; Wang, Q.; Zhou, X.; Wen, C.-y.; Yu, J.; Han, X.; Li, X.; Yan, Z.-f.; Zeng, J., A Convenient Colorimetric Method for Sensitive and Specific Detection of Cyanide Using Ag@ Au Core-Shell Nanoparticles. Sens. Actuators, B 2016, 228, 366-372.

46. Qian, L.; Yang, X., Preparation and Characterization of Ag(Au) Bimetallic Core-Shell Nanoparticles with New Seed Growth Method. Colloids Surf., A 2005, 260 (1-3), 79-85.

47. Srnová-Šloufová, I.; Lednický, F.; Gemperle, A.; Gemperlová, J., Core-Shell (Ag)Au Bimetallic Nanoparticles: Analysis of Transmission Electron Microscopy Images. Langmuir 2000, 16 (25), 9928-9935.

48. Zhang, L.; Chen, P.; Loiseau, A.; Brouri, D.; Casale, S.; Salmain, M.; Boujday, S.; Liedberg, B., Spatially Controlled Reduction and Growth of Silver in Hollow Gold Nanoshell Particles. J. Phys. Chem. C 2019, 123 (16), 10614-10621.

49. Boujday, S.; Briandet, R.; Salmain, M.; Herry, J.-M.; Marnet, P.-G.; Gautier, M.; Pradier, C.-M., Detection of Pathogenic Staphylococcus Aureus Bacteria by Gold Based Immunosensors. Microchim. Acta 2008, 163 (3-4), 203-209.

50. Ben Haddada, M.; Salmain, M.; Boujday, S., Gold Colloid-Nanostructured Surfaces for Enhanced Piezoelectric Immunosensing of Staphylococcal Enterotoxin A. Sens. Actuators, B 2018, 255, 1604-1613.

51. Zhang, L.; Salmain, M.; Liedberg, B.; Boujday, S., Naked Eye Immunosensing of Food Biotoxins Using Gold NanoparticleAntibody Bioconjugates. ACS Appl. Nano Mater. 2019, 2 (7), 4150-4158.

52. Schantz, E. J.; Roessler, W. G.; Woodburn, M. J.; Lynch, J. M.; Jacoby, H. M.; Silverman, S. J.; Gorman, J. C.; Spero, L., Purification and Some Chemical and Physical Properties of Staphylococcal Enterotoxin A. Biochemistry 1972, 11 (3), $360-366$.

53. Le Loir, Y.; Baron, F.; Gautier, M., Staphylococcus Aureus and Food Poisoning. Genet. Mol. Res. 2003, 2 (1), 63-76.

54. Salmain, M.; Ghasemi, M.; Boujday, S.; Pradier, C.-M., Elaboration of a Reusable Immunosensor for the Detection of Staphylococcal Enterotoxin A (SEA) in Milk with a Quartz Crystal Microbalance. Sens. Actuators, B 2012, 173, 148-156.

55. Slot, J. W.; Geuze, H. J., A Method to Prepare Isodisperse Colloidal Gold Sols in the Size Range 3-17 nm. Ultramicroscopy 1984, 15 (4), 383-383.

56. Bastús, N. G.; Merkoçi, F.; Piella, J.; Puntes, V., Synthesis of Highly Monodisperse Citrate-Stabilized Silver Nanoparticles of up to 200 nm: Kinetic Control and Catalytic Properties. Chem. Mater. 2014, 26 (9), 2836-2846.

57. Ma, Y.; Liang, X.; Tong, S.; Bao, G.; Ren, Q.; Dai, Z., Gold Nanoshell Nanomicelles for Potential Magnetic Resonance Imaging, Light-Triggered Drug Release, and Photothermal Therapy. Adv. Funct. Mater. 2013, 23 (7), 815-822.

58. Wang, X.; Mei, Z.; Wang, Y.; Tang, L., Gold Nanorod Biochip Functionalization by Antibody Thiolation. Talanta 2015, 136, 1-8. 
59. Wang, X.; Mei, Z.; Wang, Y.; Tang, L., Comparison of Four Methods for the Biofunctionalization of Gold Nanorods by the Introduction of Sulfhydryl Groups to Antibodies. Beilstein J. Nanotechnol. 2017, 8, 372-380.

60. Hermanson, G. T., Functional Targets. In Bioconjugate Techniques (Second Edition), Hermanson, G. T., Ed. Academic Press: New York, 2008; pp 1-168.

61. Chen, P.; Liedberg, B., Curvature of the Localized Surface Plasmon Resonance Peak. Anal. Chem. 2014, 86 (15), 7399-7405.

62. Samal, A. K.; Polavarapu, L.; Rodal-Cedeira, S.; Liz-Marzán, L. M.; Pérez-Juste, J.; Pastoriza-Santos, I., Size Tunable Au@Ag Core-Shell Nanoparticles: Synthesis and Surface-Enhanced Raman Scattering Properties. Langmuir 2013, 29 (48), $15076-15082$.

63. Link, S.; Wang, Z. L.; El-Sayed, M. A., Alloy Formation of Gold-Silver Nanoparticles and the Dependence of the Plasmon Absorption on Their Composition. J. Phys. Chem. B 1999, 103 (18), 3529-3533.

64. Zhang, L.; Hu, D.; Salmain, M.; Liedberg, B.; Boujday, S., Direct Quantification of Surface Coverage of Antibody in IgG-Gold Nanoparticles Conjugates. Talanta 2019, 204, 875-881. 


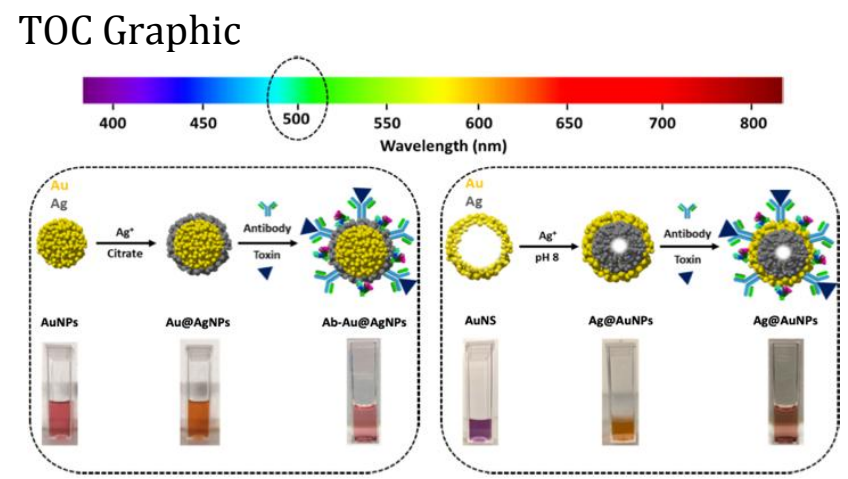

